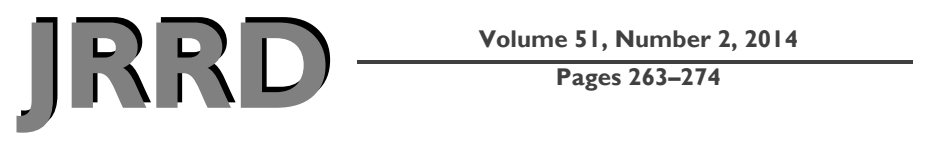

\title{
Performance-based assessment of falls risk in older veterans with executive dysfunction
}

\begin{abstract}
Barbara L. Fischer, PsyD $;^{1 *}$ William T. Hoyt, PhD ${ }^{2}$ Lawrence Maucieri, PhD ${ }^{3}$ Amy J. Kind, MD, PhD ${ }^{1,4}$ Gail Gunter-Hunt, MSW; ${ }^{1}$ Teresa Chervenka Swader, MSW; ${ }^{1}$ Ronald E. Gangnon, PhD; ${ }^{5}$ Carey E. Gleason, PhD ${ }^{1,4}$ ${ }^{1}$ Geriatric Research Education and Clinical Center, William S. Middleton Memorial Veterans Hospital, Madison, WI; ${ }^{2}$ Department of Counseling Psychology, University of Wisconsin-Madison, Madison, WI; ${ }^{3}$ Department of Psychology and Counseling, Governors State University, University Park, IL; ${ }^{4}$ Department of Medicine, School of Medicine and Public Health, University of Wisconsin-Madison, Madison, WI; ${ }^{5}$ Departments of Biostatistics and Medical Informatics and Population Health Sciences, University of Wisconsin-Madison, Madison, WI
\end{abstract}

\begin{abstract}
Falling is a serious hazard for older veterans that may lead to severe injury, loss of independence, and death. While the American Geriatrics Society (AGS) provides guidelines to screen individuals at risk for falls, the guidelines may be less successful with specific subgroups of patients. In a veteran sample, we examined whether the Timed Up and Go (TUG) test, including a modified version, the TUG-Cognition, effectively detected potential fallers whose risk was associated with cognitive deficits. Specifically, we sought to determine whether TUG tasks and AGS criteria were differentially associated with executive dysfunction, whether the TUG tasks identified potential fallers outside of those recognized by AGS criteria, and whether these tasks distinguished groups of fallers. Participants included 120 mostly male patients referred to the Memory Assessment Clinic because of cognitive impairment. TUG-Cognition scores were strongly associated with executive dysfunction and differed systematically between fallers grouped by number of falls. These findings suggest that the TUG-Cognition shows promise in identifying fallers whose risk is related to or compounded by cognitive impairment. Future research should study the predictive validity of these measures by following patients prospectively.
\end{abstract}

Key words: American Geriatrics Society falls-risk guidelines, cognitive impairment, executive function, falls, falls-risk identification, falls prevention, older adults, physical therapy, Timed Up and Go test, veterans.

\section{INTRODUCTION}

For patients, their families, and the healthcare system, reducing the rate of falls in older adults is a major priority, especially in light of data revealing that falls are the leading cause of injury deaths among older Americans [1]. Addressing falls risk is particularly challenging because falls are multifactorial in origin. According to

Abbreviations: $\mathrm{AGS}=$ American Geriatrics Society, GDS = Geriatric Depression Scale, GRECC $=$ Geriatric Research Education and Clinical Center, MAC $=$ Memory Assessment Clinic, MMSE $=$ Mini-Mental State Examination, $\mathrm{MoCA}=$ Montreal Cognitive Assessment, PCA = principal components analysis, $\mathrm{QI} / \mathrm{QA}=$ quality improvement/quality assurance, RBANS $=$ Repeatable Battery for the Assessment of Neuropsychological Status, $\mathrm{SD}=$ standard deviation, $\mathrm{TMT}=$ Trail Making Test, TUG $=$ Timed Up and Go (test), UW IRB $=$ University of Wisconsin Health Science Institutional Review Board, VA R\&D = William S. Middleton Memorial Veteran Hospital's Research and Development, VA = Department of Veterans Affairs.

*Address all correspondence to Barbara L. Fischer, PsyD; 2500 Overlook Terrace, GRECC, Wm. S. Middleton Memorial VA Hospital, Madison, WI 53705; 608-256-1901, ext 11075; fax: 608-280-7291.

Email: Barbara.fischer@va.gov

http://dx.doi.org/10.1682/JRRD.2013.03.0075 
American Geriatrics Society (AGS) guidelines [2], the most common risk factors for falls include muscle weakness, arthritis, history of falls, limited gait, balance or vision problems, use of assistive device, limited activities of daily living, depression, cognitive impairment, and age above $80 \mathrm{yr}$. While these risk factors often occur together, they are unlikely to confer equal risk in all individuals; rather, there may be subgroups of fallers for whom specific risk factors are more salient. That is, even among individuals with multiple falls risks, the strongest risk factor may be hypotension for some; for others, it may be diabetic neuropathy; while for still others, cognitive impairment may represent the most prominent risk factor.

Current guidelines established by the AGS in 2010 [2] suggest that elderly patients should be assessed for falls risk on a yearly basis. According to the recommended screening procedures used at the time the study was conducted (based on the 2001 AGS screening criteria), patients were identified as at-risk if they reported a history of two or more falls in the previous year, an acute fall (a fall for which medical attention was sought), or demonstration of gait or balance problems. It was recommended that a single self-reported fall be followed by evaluating the individual's gait and balance. The 2010 guidelines were modified to probe more specifically into the circumstances of single falls and to refer patients for falls evaluation after a self-report of gait or balance problems. Importantly, both versions of the AGS guidelines rely on patients to self-report their falls. Guidelines for both 2001 and 2010 are presented in Appendix 1 (available online only. While the AGS guidelines provide an important benchmark for determining those at risk for falls, they may not identify all subsets of at-risk patients equally well. Specifically, the guidelines may have limited efficacy in individuals with marked cognitive impairment and relatively well-preserved motor abilities. Yet patients with executive dysfunction, a specific subtype of cognitive deficit, exhibit increased risk of gait dysfunction and falls [3-7]. Moreover, many older patients may not accurately report the number of falls they have sustained [8], especially cognitively compromised individuals. Indeed, people with cognitive impairment may not remember that they have fallen, or they may not disclose their falls because of poor insight and judgment or fears of compromising their independence. For example, in one sample of older patients (cognition was not assessed), only 37.2 percent of all fallers actively disclosed their falls to healthcare providers [9]. In addi- tion, the AGS guidelines do not identify individuals at risk for falls who have not yet fallen and who are unaware of (or do not disclose) gait and balance problems. Therefore, current screening guidelines may underidentify individuals at risk for falls due to cognitive impairment.

We compared two screening methods to detect falls risk: the AGS screening criteria and the Timed Up and Go (TUG) test, a brief, simple, and established performancebased screening measure of gait and mobility [10]. Specifically, we sought to investigate the relationship between the two falls screening methods (AGS and TUG) and cognition, as well as to examine their identification of at-risk individuals. We hypothesized that, when compared with AGS criteria, TUG performance-based measures would better identify patients at risk for falls due to executive dysfunction. We also sought to discriminate among fallers using these performance-based criteria. By investigating associations between performance-based measures, executive dysfunction, and falls, we are working toward the long-term goal of developing more effective screening methods to identify cognitively impaired older patients at risk for falls so they may be targeted for appropriate clinical interventions such as physical therapy.

\section{METHODS}

The study used archival data from a quality improvement/quality assurance (QI/QA) demonstration project, the goal of which was to improve clinical ability to detect potential fallers. This QI/QA project was reviewed by the University of Wisconsin's Health Science Institutional Review Board (UW IRB), a subcommittee of the William S. Middleton Memorial Veteran Hospital's Research and Development (VA R\&D) Committee, which determined that data collection for the project was exempt research per Federal regulations. UW IRB and VA R\&D Committee approval was granted to retrospectively analyze the results.

The sample comprised 120 consecutive patients from the Geriatric Research Education and Clinical Center (GRECC) Memory Assessment Clinic (MAC) at the William S. Middleton Memorial Veterans Hospital. All participants were new MAC patients, referred because of suspected cognitive impairment as reported by the patient, family member, or primary care provider. Inclusion criteria were age $60 \mathrm{yr}$ or older; ability to walk 
20 feet without pain or risk of injury, with or without an assistive device; ability to comprehend English; and score on the Mini-Mental State Examination (MMSE) [11] of greater than 15. Exclusion criteria included inability to understand task directions.

\section{Procedures}

After check-in procedures were completed, a trained clinic nurse administered the three TUG tasks as follows. First, the TUG-Alone was performed, in which patients were asked to start from a seated position in an arm chair, rise from the chair, walk 10 feet, turn around and walk back to the chair, turn again, and sit down. Patients were asked to perform the task as quickly but as safely as they could. Two additional versions of the TUG were also administered: TUG-Manual (performing the TUG while carrying a cup of water) [12] and TUG-Cognition (performing the TUG while counting backward from 50, a variation on that used by Shumway-Cook et al. [13]). After the TUG tasks were explained and demonstrated, patients were provided one opportunity to practice the task. To ensure safety, in patients whose balance was unsteady, clinic staff fit patients with a gait belt and walked behind them lightly holding the belt for each TUG administration. Following the practice run, all participants were timed on the TUG-Alone. Next, the TUGManual task and the TUG-Cognition task were administered in randomized order, selected using a random number table. Participants who met criteria for being at risk for falls on any of the three performance-based measures were referred to physical therapy for further evaluation.

Classification of falls-risk status for the TUG-Alone and the TUG-Manual was made using cut scores established by Shumway-Cook et al. [13]. Risk status on the TUG-Cognition was determined using the 75th percentile of the interquartile range [13].

\section{Determination of Falls Status}

At their clinic visits, all patients were asked how many times they had fallen in the past year. To address the possibility of unreliable self-reporting of falls, we also compared self-reported falls with falls documented in the medical record. Since patients traditionally received their primary care through the Department of Veterans Affairs (VA) medical system, records were available to evaluate falls status. Specifically, an additional record review was undertaken for each patient, in which a clinician $(\mathrm{BF})$ reviewed notes from primary care provider visits over a 12 mo period to ascertain whether any mention of falls had been made during the previous year.

\section{Neuropsychological and Mood Measures}

After completing the walking tasks, each participant underwent neuropsychological testing, while the participants' caregivers were interviewed by social work staff. Neuropsychological tests administered are described briefly here. The MMSE is a 30 -item brief screen of global cognition that includes questions on orientation, recall, attention, working memory, language, and construction [11]. The Montreal Cognitive Assessment (MoCA) is another 30-item screening measure of global cognition assessing executive function, visuospatial construction, language, recall, verbal abstract reasoning, and orientation [14]. Serial Sevens and World Backward are tasks of working memory in which patients are asked to subtract backward from 100 by sevens and spell the word "world" backward, respectively. The Clock Draw Task assesses global cognition, executive function, and visual spatial ability [15]; individuals are asked to draw a clock with hands set to a specific time, and it is scored as either pass or fail [16]. Animal Fluency is a measure of executive function and language skills, the score representing the number of animals named in $60 \mathrm{~s}$ [17]. Trail Making Tests A and B (TMT A and TMT B) measure numeric and alphanumeric sequencing, visuospatial attention and divided attention, and speeded visual processing [18]. On these tasks, individuals are asked to connect numbers in chronological order (TMT A) or by alternating between numbers and letters in order (TMT B). Score on both TMTs represents time to complete. The Repeatable Battery for the Assessment of Neuropsychological Status (RBANS) assesses five domains of cognitive function [19]. It includes two tasks each of immediate memory (orally presented word list and story), visuospatial construction ability (line orientation and copying complex figure), language (semantic fluency and confrontation naming), attention (digit span forward and coding), and delayed memory (list learning, story, and complex figure). The Geriatric Depression Scale (GDS) (Short Form) is a 15-item self-report questionnaire of mood and depressive symptomatology [20], on which participants answered yes/no questions about their mood over the past week. One point was assigned for each item answered in the direction suggesting depressed mood. 


\section{Development of Composite Executive Function Score}

To develop a single combined measure of executive function rather than performing multiple analyses with each individual test score, we first examined Pearson product-moment correlation coefficients between individual test indices. Scores on the TMTs, representing time to complete the task, were markedly skewed. Thus, log-transformed scores were used for analyses. Because significant small to moderate correlations were found between most cognitive tests, 10 cognitive measures (RBANS coding, logTMT A, logTMT B, MoCA Total Score, Semantic Fluency, Clock Draw, RBANS Figure Copy, RBANS Digit Span, Serial Sevens, and World Backward) were subjected to a principal components analysis (PCA). Prior to performing the PCA, we assessed the suitability of the data for factor analysis. Inspection of the component matrix revealed the presence of a number of coefficients of 0.3 and above. The Kaiser-Meyer-Oklin value was established as 0.81 , exceeding the recommended value of 0.6, and Bartlett's Test of Sphericity reached statistical significance, supporting the factorability of the correlation matrix.

The PCA revealed the presence of one component with an eigenvalue exceeding one, explaining 38.17 percent of the variance. An inspection of the scree plot revealed a clear break after the first component, with the remaining components each explaining a relatively small amount of the variance. The results of this analysis supported the use of a single component solution, in the form of an attention/executive function composite score to represent this factor in further analyses. After inspection of the component matrix, tasks with loadings greater than 0.3 were included in the composite score. In the two cases in which tasks with underlying skills overlapped substantially, the measures with higher loadings in the component matrix were selected. This resulted in final selection of the following tests: RBANS Coding, logTMT A, MoCA Total Score, Semantic Fluency, Clock Draw, RBANS Figure Copy, and World Backward. Scores on each of these tasks were converted to standard ( $z$ ) scores to place them on a common metric. Because TMT A is scored in the reverse direction from the other tests (lower time reflects better cognitive functioning), scores on $\log$ TMT A were reversed before standard scores were calculated. Then, individual patients' scores on each measure were added to produce a single composite executive function score. This score was used in subsequent analyses to represent attention/executive function.

\section{Statistical Analyses}

To inform judgments about generalizability of findings, we compared TUG scores for our sample to published means by Bohannon [21] and Shumway-Cook et al. [13]. We investigated relationships between TUG tasks and tasks of attention and executive function (including the composite executive function score), and between TUG-Cognition and falls using Pearson product-moment correlation coefficients. A hierarchical linear multiple regression model controlling for age was used to test the incremental validity of the TUG-Cognition (over and above the AGS falls criteria) on performance of attention/ executive function tasks. Age was controlled for because performance on cognitive tests has been shown to decrease with age [22-23], and raw (rather than age-corrected) test scores were used in all computations in order to increase analysis uniformity and sensitivity. A Pearson correlation showed the association between past falls (as a discrete quantitative variable) and TUG-Cognition. Further, participants were divided into three falls groups: never fallers (zero falls), single fallers (one fall), and multiple fallers (two or more falls). We then created two dummy variables to compare these falls groups: high incidence versus low incidence (never or single $=0$, multiple $=1)$ and single versus never $($ never $=0$, single $=1$ ) to test for differences in TUG-Cognition scores between falls groups, using hierarchical regression models to control for age differences. Next, we examined participants with discrepant risk status on the AGS and TUG-Cognition to determine characteristics of those uniquely identified on the TUG-Cognition as at risk for falls. Finally, we examined whether identification of falls-risk status increased the referrals made to physical therapy from our MAC.

\section{RESULTS}

\section{Participant Characteristics}

The sample of 120 veterans had a mean \pm standard deviation (SD) age of $76.4 \pm 8.4$ years (range 60-90) and was overwhelmingly male (98\%). Most were in relatively good physical and emotional health as evidenced by few hospitalizations over the previous 4 mo $(15 \%)$ and a mean depression score in the nondepressed range on the GDS Short Form. Participant characteristics are presented in Table 1. 
Table 1.

Veteran characteristics. $N=120$ veterans presenting to Memory Assessment Clinic.

\begin{tabular}{|c|c|c|c|}
\hline Variable & $N^{*}$ & $\begin{array}{c}\text { Mean } \pm \text { SD or } \\
n(\%)\end{array}$ & Range \\
\hline \multicolumn{4}{|l|}{ Demographics } \\
\hline Age (yr) & 120 & $76.4 \pm 8.4$ & $60-90$ \\
\hline Sex: Male & 120 & $117(98)$ & NA \\
\hline Education (yr) & 120 & $12.3 \pm 2.6$ & $4-18$ \\
\hline Monthly Income (US\$) & 93 & $2,390.0 \pm 1,336.0$ & $470-7,056$ \\
\hline \multicolumn{4}{|l|}{ Medical History } \\
\hline Number of Psychotropic Medications & 119 & $0.5 \pm 0.8$ & $0-3$ \\
\hline History of TBI by Chart Review & 120 & $18(15)$ & NA \\
\hline History of MI by Chart Review & 120 & $11(9)$ & NA \\
\hline History of CVA by Chart Review & 120 & $17(14)$ & NA \\
\hline History of Arthritis by Chart Review & 120 & $23(19)$ & NA \\
\hline Visual Impairment as Reported in Medical Record & 114 & $35(31)$ & NA \\
\hline Number Hospitalized in Past 4 Mo by Chart Review & 120 & $18(15)$ & NA \\
\hline Number of Self-Reported Fallers & 118 & $1.0 \pm 2.0$ & $0-13$ \\
\hline Percentage of Self-Reported Fallers & 118 & $48(40)$ & - \\
\hline Number of Fallers According to Medical Record & 120 & $0.8 \pm 1.6$ & $0-10$ \\
\hline Percentage of Fallers According to Medical Record & 120 & $44(37)$ & - \\
\hline \multicolumn{4}{|l|}{ Cognitive and Mood Performance } \\
\hline MMSE & 119 & $25.2 \pm 3.1$ & $16-30$ \\
\hline $\mathrm{MoCA}$ & 63 & $18.8 \pm 5.1$ & $1-27$ \\
\hline Serial Sevens & 100 & $3.3 \pm 1.6$ & $0-5$ \\
\hline World Backward & 118 & $4.1 \pm 1.2$ & $0-5$ \\
\hline RBANS Coding (timed) & 114 & $24.4 \pm 10.3$ & $3-48$ \\
\hline RBANS Digit Span & 117 & $8.6 \pm 2.2$ & $4-15$ \\
\hline RBANS Figure Copy ${ }^{\dagger}$ & 116 & $15.3 \pm 3.7$ & $2-20$ \\
\hline RBANS Fluency $^{\dagger}$ (timed) & 119 & $11.9 \pm 4.8$ & $2-23$ \\
\hline Semantic Fluency (timed) & 106 & $13.6 \pm 5.4$ & $1-31$ \\
\hline Clock Draw & 120 & $0.5 \pm 0.50$ & $0-1$ \\
\hline TMT A $\$$ (score in seconds) & 114 & $66.2 \pm 44.1$ & $20-339$ \\
\hline TMT B ${ }^{\dagger}$ (score in seconds) & 117 & $274.3 \pm 194.4$ & $46-1,383$ \\
\hline Score on Depression Screening Measure ${ }^{\S}$ & 118 & $3.7 \pm 3.2$ & $0-13$ \\
\hline \multicolumn{4}{|c|}{$\begin{array}{l}{ }^{*} N \text { varied slightly depending on time constraints. Some patients worked too slowly to complete all cognitive measures and selected tests were omitted based on clin- } \\
\text { ical judgment. Of note, substantially lower number of patients completing MoCA reflects its introduction to clinic midway through study. } \\
\text { tDuff et al. [29] values include mean } \pm \text { SD for } 76 \text { yr old patients. } \\
\$ \text { Tombaugh [30] values include mean } \pm \text { SD for } 76 \text { yr old patients. } \\
{ }^{\$} \text { Depression measured with Geriatric Depression Scale-Short Form. Scale }=0-15 ; 0-5 \text { points indicating no or minimal symptoms, } 6-10 \text { points suggesting mild to } \\
\text { moderate depressive symptoms, and } 11-15 \text { points consistent with moderate to severe depressive symptoms. } \\
\text { CVA = cerebral vascular accident (stroke), MI = myocardial infarction (heart attack), MMSE = Mini-Mental State Examination, MoCA }=\text { Montreal Cognitive } \\
\text { Assessment, NA = not applicable, RBANS = Repeatable Battery for the Assessment of Neurocognitive Status, SD = standard deviation, TBI = traumatic brain } \\
\text { injury, TMT = Trail Making Test. }\end{array}$} \\
\hline
\end{tabular}

TUG task times and interquartile ranges from the present study as well as from Bohannon [21] and ShumwayCook et al.'s [13] results are presented in Table 2. Independent sample $t$-tests comparing TUG task scores from all three studies revealed significant differences. Veterans in the present sample performed the TUG-Alone significantly more slowly (mean $\pm \mathrm{SD}=13.35 \pm 6.02$ ) than did individuals in Bohannon's analysis of normal adults in both the 60 to $69 \mathrm{yr}$ old range $($ mean $=8.1, t(294)=7.17$, $p<0.001)$ and the 70 to $79 \mathrm{yr}$ old range (mean $=9.2$, $t(916)=3.17, p=0.002$ ) (two-tailed). Of interest, the present sample was not significantly different from Bohannon's group of individuals in the 80 to $99 \mathrm{yr}$ range (mean = 11.3, $t(1,220)=0.98, p=0.32)($ two-tailed $)$, indicating 
JRRD, Volume 51, Number 2, 2014

Table 2.

Performance on TUG tasks; data from three studies.

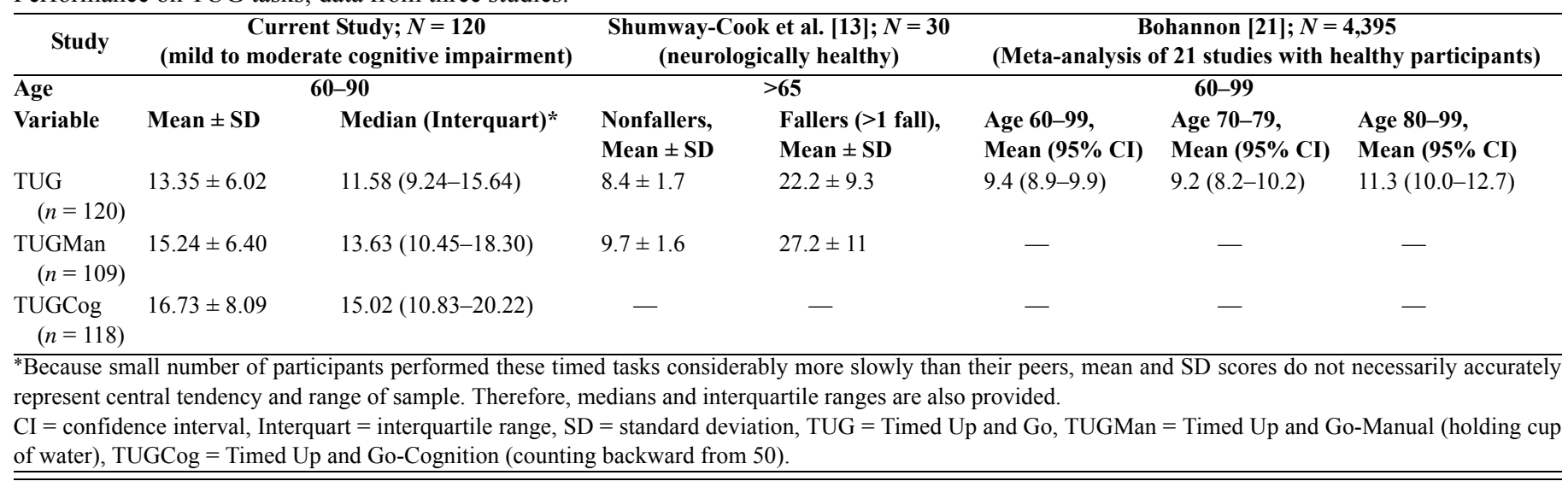

that our sample performed similarly to healthy individuals of more advanced age. Significant differences were also found between TUG-Alone times in the present sample and Shumway-Cook et al.'s scores for nonfallers (mean \pm $\mathrm{SD}=8.4 \pm 1.7, t(133)=3.16, p=0.002)($ two-tailed $)$ and for fallers (mean $\pm \mathrm{SD}=22.2 \pm 9.3, t(133)=-5.01, p<$ 0.001 ) (two-tailed), with this sample of veterans performing the task significantly more slowly.

Independent sample $t$-tests comparing scores on the TUG-Manual in the present sample (mean $\pm \mathrm{SD}=15.24 \pm$ 6.40) with Shumway Cook et al.'s [13] scores on the same task (participants were $65 \mathrm{yr}$ of age or older) revealed significant differences between published data from nonfallers $($ mean $\pm \mathrm{SD}=9.7 \pm 1.6, t(122)=43.33, p=0.001)$ (two-tailed) and fallers (mean $\pm \mathrm{SD}=27.2 \pm 11, t(122)=$ $-6.13, p<0.001)$ (two-tailed). Because the TUG-Cognition task has not been used in other studies, comparisons to other samples could not be conducted.

\section{Correlation of Attention/Executive Scores with TUG Task Parameters}

Bivariate correlations between measures of attention and executive function and three TUG task parameters are presented in Appendix 2 (available online only). Relative to the TUG-Alone and the TUG-Manual, the TUG-Cognition demonstrated the strongest correlations with individual measures of attention and executive function. Correlations between the attention/executive function composite score and the TUG-Alone $(r=-0.36)$, the TUG-Manual $(r=-$ $0.28)$, and the TUG-Cognition $(r=-0.39)$ further reflected the TUG-Cognition's robust relationship with cognitive function.

\section{Regression Analyses Predicting Attention/Executive Function: Comparison of Performance-Based Measures and AGS Criteria}

Hierarchical multiple regression analyses are reported in Table 3 and suggest that TUG-Cognition accounts for significant variance in attention/executive function composite scores, after controlling for both age and AGS risk status. Age was entered at Step 1, explaining 10 percent of the variance in executive functioning. After the entry of the dichotomous variable "Meets AGS Criteria," the model explained 15 percent of the total variance, with the significance test for the change in the coefficient of determination indicating that AGS accounted for a significant amount of variance in executive functioning after controlling for age. When TUGCognition was entered into the model, the total variance in executive functioning explained was 20 percent, again a significant gain in variance explained. In the final model, age, AGS criteria, and TUG-Cognition emerged as statistically significant, with age recording the highest beta value followed by TUG-Cognition.

\section{Relationship Between Falls and TUG Tasks}

The Pearson correlation between the number of falls reported at the MAC visit and TUG-Cognition was not statistically significant $(r=0.12, p=0.20)$. However, TUG-Cognition tasks demonstrated moderate correlations with falls documented in the medical record $(r=0.27, p=$ 0.003 ), establishing a significant relationship between prior falls and TUG scores. Dot plots of TUG-Cognition scores compared with falls in the medical record revealed three distinct groups of scores: never fallers (zero falls), single fallers (one fall), and multiple fallers (two or more 
Table 3.

Hierarchical regression analysis of AGS and TUG-Cognition predicting composite executive functioning after controlling for age.

\begin{tabular}{lccccccc}
\hline \multicolumn{1}{c}{ Entered } & $\boldsymbol{\beta}$ & $\boldsymbol{R}^{\mathbf{2}}$ & $\boldsymbol{R}^{\mathbf{2}}$ Change & $\boldsymbol{F}$ change & $\boldsymbol{d} \boldsymbol{f}_{\mathbf{1}}$ & $\boldsymbol{d} \boldsymbol{f}_{\mathbf{2}}$ & $\boldsymbol{p}$-Value \\
\hline 1. Age & -0.31 & 0.10 & 0.10 & 12.53 & 1 & 116 & 0.001 \\
2. AGS & -0.24 & 0.15 & 0.06 & 7.47 & 1 & 115 & 0.007 \\
3. TUG-Cognition & -0.26 & 0.20 & 0.05 & 6.71 & 1 & 114
\end{tabular}

Note: AGS $=$ meets AGS criteria for falls risk $(1=$ yes; $0=$ no $)$.

AGS $=$ American Geriatrics Society, $d f=$ degrees of freedom, TUG-Cognition = Timed Up and Go-Cognition .

falls). The Figure depicts the relationship between falls and TUG-Cognition scores.

Hierarchical regression analysis using dummy variables to represent differences in falls incidence (high versus low, single versus never) revealed significant differences in TUG-Cognition scores. Age was entered at Step 1, explaining 17.8 percent of the total model variance $(F(1,116)=25.14, p<0.001)$. After entry of the two variables comparing falls groups at Step 2, the total variance explained by the model as a whole was 27.7 percent $(F(2,114)=7.76, p=0.001)$. These two variables explained an additional 10 percent of the variance in TUG-Cognition after controlling for age, $\left(R^{2}\right.$ change $=$ $0.10, F$ change $(3,114)=14.53, p<0.001)$. In the final model, only the comparison between high versus low incidence (nonfallers + single fallers and multiple fallers) was statistically significant $(\beta=3.72, p=0.03)$, indicating that those in the multiple falls group required an addi-

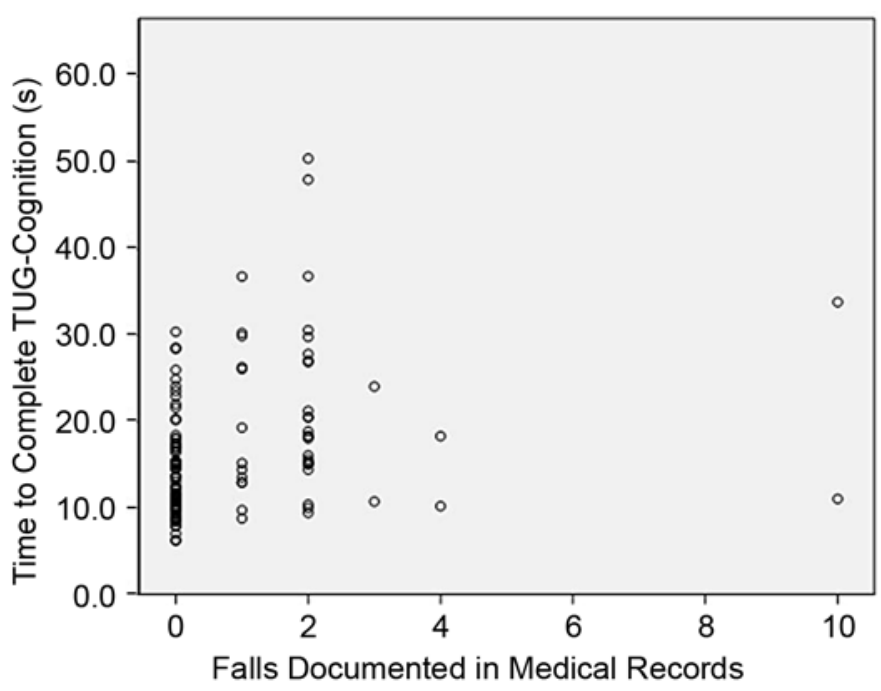

Figure.

Scatter plot of Timed Up and Go-Cognition scores compared with falls documented in medical record. tional $3.72 \mathrm{~s}$ to complete the TUG-Cognition than those in the never and single fallers groups.

\section{Descriptive Data for Individuals with Discordant Risk Status as Defined by AGS Criteria and TUG-Cognition}

Analysis of discrepant risk status on falls-risk measures revealed 14 veterans uniquely identified as at risk for falls by the TUG-Cognition and 21 veterans uniquely identified as at-risk by AGS criteria. The individuals uniquely identified by the TUG-Cognition task were significantly older (mean difference of $6 \mathrm{yr} ; p=0.007$ ) than those solely identified by AGS. In addition, in the previous 4 mo, significantly fewer individuals identified by TUG-Cognition had been hospitalized compared with those identified by AGS criteria $(p=0.01)$, suggesting a lower level of frailty in patients whose risk was identified by TUG-Cognition. Finally, the group uniquely identified by the TUG-Cognition included three times the rate of mild cognitive impairment diagnoses compared with the group labeled at-risk by AGS criteria, although this difference did not reach statistical significance in this relatively small sample $(p=0.14)$. In summary, the group uniquely identified by the TUG-Cognition was older, less frail, and exhibited a trend toward more mild forms of cognitive impairment than those in the group uniquely identified by the AGS criteria.

\section{Increased Referrals to Physical Therapy}

Finally, records obtained from physical therapy service indicated that referral numbers from the MAC more than doubled in frequency after the TUG-Cognition was implemented. For example, there was an average of one referral per month in 2007 and more than two referrals per month by 2010. These data suggest that use of the TUG-Cognition may facilitate further evaluation of veterans with questionable mobility status. 


\section{DISCUSSION}

This study compared two methods of identifying individuals at risk for falls (as documented in the medical record): the AGS criteria and two well-established TUG performance-based measures, the TUG-Alone and the TUG-Manual, as well as a modified version of the test, the TUG-Cognition. Results suggested that while both the AGS and TUG falls-risk criteria (all three forms) were associated with cognitive impairment, performance on the TUG-Cognition predicted variance in attention/executive functioning over and above that accounted for by the AGS falls-risk criteria. Moreover, the TUG-Cognition successfully discriminated between never/single fallers and multiple fallers. Finally, the TUG-Cognition may be sensitive to falls risk in a subgroup of patients not detected using the AGS criteria. Those uniquely identified by the TUG-Cognition were older and less physically frail, with a trend toward increased rates of mild cognitive impairment than those identified solely by the AGS criteria. Altogether, these analyses suggested that the TUG tasks, particularly the TUG-Cognition, are a necessary supplement to the widely used AGS criteria for older veterans with cognitive impairment. By relying on performance rather than recall of recent falls, the TUGCognition can more effectively identify individuals whose falls risk is associated with cognitive impairment.

\section{Performance-Based Measures Detect Falls Risk Associated with Attention/Executive Function}

After controlling for age and risk status as defined by the AGS criteria, the TUG-Cognition demonstrated relationships with attention/executive function tasks. Numerous studies have confirmed the relationship between executive dysfunction and falls. The ability to maneuver in complex environments depends on intact attention/ executive function, and deficits in these areas represent risk factors for gait dysfunction and falls [3-7]. This may be especially true in patient populations with neurological disorders, in whom gait patterns may already be subtly altered [24]. Moreover, failure to appropriately identify patients who are at risk for falling likely results in a corresponding failure to implement necessary intervention strategies. Patients may persist in engaging in hazardous activities or maintain exposure to suboptimal environments because they overestimate their abilities or are unaware of the risk to their safety [25]. Altogether, these data emphasize the importance of using perfor- mance-based assessments for patients whose risk for falls is associated with executive dysfunction.

The present data are consistent with recent findings by Herman et al. [26], revealing correlations between the TUG, executive function, and multiple falls, whereas two other measures of mobility, the Berg Balance Test and the Dynamic Gait Index, did not show significant correlations with either cognition or falls. While future studies will help elucidate the relationships between measures of falls risk, cognition, and prospective falls, it is clear that both gait and executive dysfunction constitute falls-risk factors and opportunities for intervention.

Of note, self-reported falls and falls reported in the medical record differed in this study. Because patients with cognitive impairment may incorrectly report their falls, it is suggested that falls reported in the medical record may more accurately represent actual falls. We speculate that compared with the report on falls gathered during a typical appointment check-in process, the medical record may document falls temporally closer to their occurrence; i.e., the report is less affected by impaired recall than a report based on a patient's recall of falls occurring in the past year. This may be especially true for individuals with cognitive impairment. In this study, falls reported in the medical record (but not self-reported falls) were significantly related to TUG performance. Because the AGS criteria depend on self-report, the TUG-Cognition may provide a useful supplement to the AGS-based risk assessment, especially among patients with impaired cognition.

\section{TUG Tasks Distinguished Between Infrequent Fallers and Recurrent Fallers}

The TUG-Cognition distinguished between two subgroups of fallers, specifically, never and single fallers and multiple fallers. In other words, multiple fallers demonstrated significantly worse performance on TUG-Cognition than single fallers and never fallers, supporting the idea that multiple fallers are more likely to fall because of enduring underlying difficulties, including cognitive impairment. Whereas a single fall may reflect nothing more than unfortunate circumstances, multiple falls are more likely to signal a breakdown in complex walking physiology or neuromuscular circuitry. The additional cognitive load inherent in counting backward (TUG-Cognition) was sufficient to significantly alter the performance of those people for whom the mechanics of walking were already compromised. 


\section{TUG Tasks and AGS Criteria Identified Unique Groups of Potential Fallers}

The group uniquely identified as at-risk by the TUGCognition included individuals in better physical health, with a trend toward higher rates of mild cognitive impairment than those identified solely by AGS criteria. As such, TUG-Cognition may be sensitive to more subtle cognitive dysfunction than the AGS criteria, and it may be more adept at detecting falls risk due to cognitive dysfunction earlier in the neurodegenerative process. From a clinical standpoint, this is important because it suggests that early determination of falls risk due to cognitive impairment can be ascertained without a full neuropsychological evaluation. Administration of the TUG tasks can be completed in 5 min in a primary care setting, and they can provide a determination of falls risk sufficient to refer for further evaluation if necessary. Screening results could facilitate earlier referrals for intervention and the generation of falls-prevention strategies while patients are best able to operationalize and benefit from them.

\section{TUG-Cognition Shows Promise as Means of Screen- ing Fallers with Cognitive Impairment}

The TUG-Alone and TUG-Manual are well-validated measures of falls risk [10,12,21,26-28]. The current data suggest that a modified version of the TUG, the TUGCognition, shows promise in identifying patients at risk for falls due to cognitive impairment, including those who might not be detected by AGS criteria. Not only was the TUG-Cognition significantly correlated with tests of attention and executive function (Appendix 2, available online only), it also effectively discriminated between falls groups in this cross-sectional analysis. These results suggest that the addition of the cognitive challenge (counting backward from 50 by ones) achieved the desired result: producing TUG scores that index executive functioning as well as mobility. We speculate that the TUG-Cognition may identify individuals with even mild levels of cognitive decline and may differentially identify individuals with similar scores on cognitive screening tests (e.g., the MMSE) based on their level of executive function. An important goal for future research includes a prospective study of falls risk using the TUG-Cognition. With a sufficient sample size, cut scores can be established (using receiver operant characteristics curves) that optimize predictive accuracy. While the AGS guidelines remain the standard for falls risk, the TUG-Cognition is proposed as a complement to AGS screening procedures and should be especially useful in cases in which cognitive impairment is contributing to risk.

\section{TUG Measures Increased Referrals to Physical Therapy}

Finally, as an incidental finding, it is noted that performance on the TUG measures generated more than double the rate of referrals to physical therapy from the VA MAC during the period of project implementation than the AGS criteria had in the 6 mo prior to the beginning of the project. Although cognitive impairment is a risk factor for falls, it is not practicable to conduct a comprehensive falls evaluation on every individual who demonstrates cognitive deficits. At the same time, because the consequences of falling are so great, we wished to avoid allowing any potentially at-risk patient to "slip through the cracks" through lack of detection. By using performance-based measures, we were able to identify cognitively impaired individuals at higher risk for falls and to provide them with a more comprehensive evaluation through physical therapy. While an analysis of actual falls prevented as a result of this intervention was beyond the scope of this study, future research should longitudinally assess the relationship of identification of at-risk status by the TUG-Cognition, referral to physical therapy, and future falls.

\section{Limitations}

This study presents with some limitations. The majority of participants were male, so validity for female patients remains to be tested. However, because the veteran population increasingly includes women, we wished to ensure that our sample was representative of the veterans presenting to the MAC. In addition, the veteran population may have unique medical and demographic characteristics, also limiting generalizability of the findings to nonveteran populations. Nevertheless, a comparison of our data with other published TUG times suggests that our results are indeed generalizable to other samples. The cross-sectional single group design is less informative than a longitudinal design, and falls were recorded retrospectively rather than prospectively. Thus, the degree to which the TUG-Cognition prospectively predicts falls is unknown and is an important goal for future research. The chart review was conducted by a single clinician (BF) in a single medical system. In cases of ambiguous diagnosis, this could lead to the possibility of rater error or bias. Additionally, falls that resulted in treatment outside the VA system and were not reported to 
a VA clinician may have been omitted. However, diagnoses in clinic were based on discussion occurring in multidisciplinary conferences, during which all clinical data are reviewed by the team of clinicians. Analyses may have been affected by the non-normal distribution of several variables; this may have constrained or attenuated results. Finally, the study was performed before the AGS falls-risk criteria were revised, using slightly different criteria for falls risk. However, the revised AGS criteria remain dependent on patient recall of falls to establish falls risk, creating a clear distinction with the performance-based TUG tasks.

\section{CONCLUSIONS}

This study compared two falls-screening measures in a sample of older veterans. Findings suggested that cognitive impairment predicted falls-risk status defined by both the TUG tasks and AGS criteria; however, TUGCognition accounted for additional variance beyond that predicted by AGS. As such, the TUG-Cognition may be more effective in identifying individuals at risk for falls due to executive dysfunction than the AGS screening criteria. For clinicians seeking to identify falls risk associated with cognitive dysfunction, these data highlight the need to use performance-based measures. Moreover, using the TUG-Cognition to detect individuals at-risk due to compromised executive function ability increases the possibility of intervening more quickly and efficaciously in this subgroup of fallers. This is critical because individuals with mild cognitive deficits may still benefit considerably from physical therapy and other cognitively based strategies, thereby preventing future injurious falls. Overall, identifying patients who are at risk for future falls and providing effective intervention as early as possible will help avoid significant suffering and lower healthcare costs, while facilitating retention of functional independence and better quality of life.

\section{ACKNOWLEDGMENTS}

\author{
Author Contributions: \\ Study conceptualization and design: B. L. Fischer, C. E. Gleason. \\ Acquisition of data: B. L. Fischer, A. J. Kind, G. Gunter-Hunt, \\ T. C. Swader. \\ Analysis and interpretation of data: B. L. Fischer, W. T. Hoyt, \\ L. Maucieri, C. E. Gleason.
}

Manuscript preparation: B. L. Fischer, W. T. Hoyt, L. Maucieri, A. J. Kind, G. Gunter-Hunt, T. C. Swader, C. E. Gleason, R. E. Gangnon. Consultation: R. E. Gangnon.

Financial Disclosures: The authors have declared that no competing interests exist.

Funding/Support: Dr. Gleason's time was supported by the Wisconsin Alzheimer Disease Research Center. Dr. Fischer's time was supported by a VA GRECC Advanced Fellowship.

Institutional Review: This study used archival data from a QI/QA demonstration project whose goal was to improve clinical ability to detect potential fallers. This QI/QA project was reviewed by the UW IRB), a subcommittee of the VA R\&D Committee, which determined that data collection for the project was exempt research per Federal regulations. UW IRB and VA R\&D Committee approval was granted to retrospectively analyze the results.

\section{REFERENCES}

1. National Safety Council. Injury Facts. 2008 ed. Itasca (IL): National Safety Council; 2008.

2. Panel on Prevention of Falls in Older Persons, American Geriatrics Society and British Geriatrics Society. Summary of the updated American Geriatrics Society/British Geriatrics Society clinical practice guideline for prevention of falls in older persons. J Am Geriatr Soc. 2011;59(1):148-57. [PMID:21226685] http://dx.doi.org/10.1111/j.1532-5415.2010.03234.x

3. Faulkner KA, Redfern MS, Cauley JA, Landsittel DP, Studenski SA, Rosano C, Simonsick EM, Harris TB, Shorr RI, Ayonayon HN, Newman AB; Health, Aging, and Body Composition Study. Multitasking: Association between poorer performance and a history of recurrent falls. J Am Geriatr Soc. 2007;55(4):570-76. [PMID:17397436] http://dx.doi.org/10.1111/j.1532-5415.2007.01147.x

4. Holtzer R, Friedman R, Lipton RB, Katz M, Xue X, Verghese $\mathrm{J}$. The relationship between specific cognitive functions and falls in aging. Neuropsychology. 2007;21(5): 540-48. [PMID:17784802] http://dx.doi.org/10.1037/0894-4105.21.5.540

5. Pettersson AF, Olsson E, Wahlund LO. Effect of divided attention on gait in subjects with and without cognitive impairment. J Geriatr Psychiatry Neurol. 2007;20(1):58-62. [PMID:17341772] http://dx.doi.org/10.1177/0891988706293528

6. Springer S, Giladi N, Peretz C, Yogev G, Simon ES, Hausdorff JM. Dual-tasking effects on gait variability: The role of aging, falls, and executive function. Mov Disord. 2006; 21(7):950-57. [PMID:16541455] http://dx.doi.org/10.1002/mds.20848

7. Yogev-Seligmann G, Hausdorff JM, Giladi N. The role of executive function and attention in gait. Mov Disord. 
2008;23(3):329-42, quiz 472. [PMID:18058946]

http://dx.doi.org/10.1002/mds.21720

8. Mackenzie L, Byles J, D’Este C. Validation of self-reported fall events in intervention studies. Clin Rehabil. 2006; 20(4):331-39. [PMID:16719031] http://dx.doi.org/10.1191/0269215506cr947oa

9. Collerton J, Kingston A, Bond J, Davies K, Eccles MP, Jagger C, Kirkwood TB, Newton JL. The personal and health service impact of falls in 85 year olds: Cross-sectional findings from the Newcastle $85+$ cohort study. PLoS ONE. 2012;7(3):e33078. [PMID:22427954] http://dx.doi.org/10.1371/journal.pone.0033078

10. Podsiadlo D, Richardson S. The timed "Up \& Go": A test of basic functional mobility for frail elderly persons. J Am Geriatr Soc. 1991;39(2):142-48. [PMID:1991946]

11. Folstein MF, Folstein SE, McHugh PR. "Mini-mental state." A practical method for grading the cognitive state of patients for the clinician. J Psychiatr Res. 1975;12(3):89-98. [PMID:1202204] http://dx.doi.org/10.1016/0022-3956(75)90026-6

12. Lundin-Olsson L, Nyberg L, Gustafson Y. Attention, frailty, and falls: The effect of a manual task on basic mobility. J Am Geriatr Soc. 1998;46(6):758-61. [PMID:9625194]

13. Shumway-Cook A, Brauer S, Woollacott M. Predicting the probability for falls in community-dwelling older adults using the Timed Up \& Go Test. Phys Ther. 2000;80(9): 896-903. [PMID:10960937]

14. Nasreddine ZS, Phillips NA, Bédirian V, Charbonneau S, Whitehead V, Collin I, Cummings JL, Chertkow H. The Montreal Cognitive Assessment, MoCA: A brief screening tool for mild cognitive impairment. J Am Geriatr Soc. 2005;53(4):695-99. [PMID:15817019] http://dx.doi.org/10.1111/j.1532-5415.2005.53221.x

15. Goodglass HE. Assessment of aphasia and related disorders. Philadelphia (PA): Lea \& Febiger; 1972.

16. Scanlan JM, Brush M, Quijano C, Borson S. Comparing clock tests for dementia screening: Naïve judgments vs formal systems - what is optimal? Int J Geriatr Psychiatry. 2002;17(1):14-21. [PMID:11802225]

http://dx.doi.org/10.1002/gps.516

17. Strauss E, Sherman E, Spreen O. A compendium of neuropsychological tests: Administration, norms, and commentary. 3rd ed. Oxford (UK): Oxford University Press; 2006. p. 1216.

18. Partington JE. R. G. Partington's pathway test. Psychological Services Center Bulletin. 1949;1:9-20.

19. Randolph C. Repeatable Battery for the Assessment of Neuropsychological Status manual. San Antonio (TX): The Psychological Corporation; 1998.

20. Fountoulakis KN, Tsolaki M, Iacovides A, Yesavage J, O'Hara R, Kazis A, Ierodiakonou C. The validation of the short form of the Geriatric Depression Scale (GDS) in Greece. Aging (Milano). 1999;11(6):367-72.

[PMID:10738851]

21. Bohannon RW. Reference values for the timed up and go test: A descriptive meta-analysis. J Geriatr Phys Ther. 2006;29(2):64-68. [PMID:16914068] http://dx.doi.org/10.1519/00139143-200608000-00004

22. Oh H, Madison C, Haight TJ, Markley C, Jagust WJ. Effects of age and $\beta$-amyloid on cognitive changes in normal elderly people. Neurobiol Aging. 2012;33(12):2746-55. [PMID:22429886] http://dx.doi.org/10.1016/j.neurobiolaging.2012.02.008

23. Kerchner GA, Racine CA, Hale S, Wilheim R, Laluz V, Miller BL, Kramer JH. Cognitive processing speed in older adults: Relationship with white matter integrity. PLoS ONE. 2012;7(11):e50425. [PMID:23185621] http://dx.doi.org/10.1371/journal.pone.0050425

24. Scherder E, Eggermont L, Swaab D, van Heuvelen M, Kamsma Y, de Greef M, van Wijck R, Mulder T. Gait in ageing and associated dementias; its relationship with cognition. Neurosci Biobehav Rev. 2007;31(4):485-97.

[PMID:17306372]

http://dx.doi.org/10.1016/j.neubiorev.2006.11.007

25. Lafargue G, Noël M, Luyat M. In the elderly, failure to update internal models leads to over-optimistic predictions about upcoming actions. PLoS ONE. 2013;8(1):e51218.

[PMID:23326312]

http://dx.doi.org/10.1371/journal.pone.0051218

26. Herman T, Giladi N, Hausdorff JM. Properties of the 'timed up and go' test: More than meets the eye. Gerontology. 2011;57(3):203-10. [PMID:20484884]

http://dx.doi.org/10.1159/000314963

27. Viccaro LJ, Perera S, Studenski SA. Is timed up and go better than gait speed in predicting health, function, and falls in older adults? J Am Geriatr Soc. 2011;59(5):887-92. [PMID:21410448] http://dx.doi.org/10.1111/j.1532-5415.2011.03336.x

28. Zhu K, Devine A, Lewis JR, Dhaliwal SS, Prince RL. "Timed up and go" test and bone mineral density measurement for fracture prediction. Arch Intern Med. 2011; 171(18):1655-61. [PMID:21987195] http://dx.doi.org/10.1001/archinternmed.2011.434

29. Duff K, Patton D, Schoenberg MR, Mold J, Scott JG, Adams RL. Age- and education-corrected independent normative data for the RBANS in a community dwelling elderly sample. Clin Neuropsychol. 2003;17(3):351-66. [PMID:14704885] http://dx.doi.org/10.1076/clin.17.3.351.18082

30. Tombaugh TN. Trail Making Test A and B: Normative data stratified by age and education. Arch Clin Neuropsychol. 2004;19(2):203-14. [PMID:15010086] http://dx.doi.org/10.1016/S0887-6177(03)00039-8 
JRRD, Volume 51, Number 2, 2014

Submitted for publication March 26, 2013. Accepted in revised form August 28, 2013.

This article and any supplemental material should be cited as follows:

Fischer BL, Hoyt WT, Maucieri L, Kind AJ, Gunter-Hunt G, Swader TC, Gangnon RE, Gleason CE. Performancebased assessment of falls risk in older veterans with executive dysfunction. J Rehabil Res Dev. 2014;51(2): 263-74.

http://dx.doi.org/10.1682/JRRD.2013.03.0075

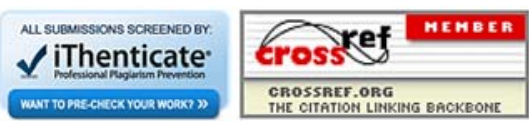

